\title{
Transsynaptic EphB/Ephrin-B Signaling Regulates Growth of Presynaptic Boutons Required for Classical Conditioning
}

\author{
Wei Li, Zhaoqing Zheng, and Joyce Keifer \\ Neuroscience Group, Division of Basic Biomedical Sciences, University of South Dakota Sanford School of Medicine, Vermillion, South Dakota, 57069
}

Learning-related presynaptic remodeling has been documented in only a few systems, and its molecular mechanisms are largely unknown. Here we describe a role for the bidirectional EphB/ephrin-B signaling system in structural plasticity of presynaptic nerve terminals using an in vitro model of classical conditioning. Conditioning or BDNF application induced significant growth of auditory nerve presynaptic boutons that convey the conditioned stimulus to abducens motor neurons. Interestingly, bouton enlargement occurred only for those synapses apposed to motor neuron dendrites rather than to somata. Phosphorylation of ephrin-B1, but not EphB2, was induced by both conditioning and BDNF application and was inhibited by postsynaptic injections of ephrin-B antibody. Finally, suppression of postsynaptic ephrin-B function inhibited presynaptic bouton enlargement that was rescued by activation of EphB2 by ephrin-B1-Fc. These data provide evidence for ephrin-B-induced EphB2 forward signaling in presynaptic structural plasticity during classical conditioning. They also reveal a functional interaction between BDNF/TrkB and the Eph/ephrin signaling systems in the coordination of presynaptic and postsynaptic modifications during conditioning.

\section{Introduction}

Activity-dependent synaptic modifications involve structural changes in neuronal connections that occur as large-scale pathway reorganization or selective synaptic remodeling (Holtmaat and Svoboda, 2009). Long-term potentiation (LTP), an established model of learning and memory, induces emergence and stabilization of new dendritic spines (Holtmaat et al., 2006; De Roo et al., 2008; Yang et al., 2008), while its counterpart, longterm depression (LTD), results in loss of spines (Zhou et al., 2004; Becker et al., 2008). Synaptic plasticity and learning may also be accompanied by enlargement of the postsynaptic density (PSD) and formation of multiple synapse boutons (Geinisman et al., 2000, 2001). Notably, two-photon microscopy has shown realtime spine growth during LTP (De Roo et al., 2008; Yang et al., 2008). These studies have largely focused on postsynaptic structural modifications, but learning-related presynaptic remodeling is not well characterized.

Molecular signals such as growth factors and cell adhesion molecules involved in formation of synaptic specializations during development have been implicated in synaptic plasticity (Lai and Ip, 2009; Cohen-Cory et al., 2010). Brain-derived neu-

Received Dec. 6, 2010; revised April 20, 2011; accepted April 26, 2011.

Author contributions: J.K. designed research;W.L. and Z.Z. performed research;W.L., Z.Z., and J.K. analyzed data; J.K. and W.L. wrote the paper.

This work was supported by National Institutes of Health Grants NS051187 and P20 RR015567, which supports a Center of Biomedical Research Excellence, to J.K. We thank Dr. Frances Day for assistance with the confocal microscopy.

Correspondence should be addressed to Dr. Joyce Keifer, Neuroscience Group, Basic Biomedical Sciences, University of South Dakota Sanford School of Medicine, 414 East Clark Street, Vermillion, SD 57069. E-mail: jkeifer@usd.edu.

W. Li's present address: Department of Neurobiology, University of Alabama at Birmingham, Birmingham, AL 35294.

DOI:10.1523/JNEUROSCI.6343-10.2011

Copyright $\odot 2011$ the authors $\quad 0270-6474 / 11 / 318441-09 \$ 15.00 / 0$ rotrophic factor (BDNF), for example, induces axonal branching, dendritic outgrowth, and synapse formation. Time-lapse imaging has shown that increased spine size after focal uncaging of glutamate was blocked by inhibitors of BDNF signaling, providing strong evidence for BDNF in structural plasticity (Tanaka et al., 2008). In contrast to BDNF, the cell adhesion molecules Eph/ephrin are tethered to cell membranes (Klein, 2009; Lai and Ip, 2009). The Eph/ephrin signaling system is unique in that both may act as receptor and ligand and can be localized presynaptically or postsynaptically. Signaling proceeds in forward or reverse directions, or bidirectionally. Transfection of postsynaptic neurons with EphB2 lacking the ephrin-binding domain was shown to reduce presynaptic differentiation and synaptic transmission (Kayser et al., 2006; Lim et al., 2008). Presynaptically expressed LTP at mossy fiber-CA3 synapses was also impeded by postsynaptic application of antibodies against EphB (Contractor et al., 2002). While progress has been made on the function of BDNF and Eph/ephrin individually, little is known about their interactions.

In this study, we used an in vitro model of eyeblink classical conditioning in which stimulation of the auditory [the "tone" conditioned stimulus (CS)] and trigeminal [the "airpuff" unconditioned stimulus (US)] nerves was paired to generate conditioned responses (CRs) characteristic of eyeblinks recorded from the abducens nerve (Keifer and Zheng, 2010). Expression of synaptic plasticity during conditioning involves the delivery of postsynaptic GluR1 and GluR4 AMPAR subunits in which BDNF has a pivotal role (Li and Keifer, 2008, 2009; Keifer et al., 2009). Here, we show that conditioning or BDNF application results in rapid growth of auditory nerve presynaptic boutons apposed specifically to dendrites but not somata of abducens motor neurons. Inhibition of postsynaptic ephrin-B function by localized antibody injection blocks bouton growth and CR acquisition while suppression of bouton growth is rescued by the EphB2 activator 
ephrin-B1-Fc. These data support a role for postsynaptic ephrinB-induced EphB2 forward signaling in presynaptic structural plasticity during classical conditioning.

\section{Materials and Methods}

Training procedures. Freshwater pond turtles, Trachemys scripta elegans, of either sex and purchased from commercial suppliers were anesthetized by hypothermia until torpid and decapitated. All experiments involving the use of animals were performed in accordance with the guidelines of the National Institutes of Health and the Institutional Animal Care and Use Committee. The brainstem was transected at the levels of trochlear and glossopharyngeal nerves and the cerebellum was removed as described previously (Anderson and Keifer, 1999). The brainstem was continuously bathed $(2-4 \mathrm{ml} / \mathrm{min})$ with physiological saline containing the following (in mM): $100 \mathrm{NaCl}, 6 \mathrm{KCl}, 40 \mathrm{NaHCO}_{3}, 2.6 \mathrm{CaCl}_{2}, 1.6 \mathrm{MgCl}_{2}$, and 20 glucose, which was oxygenated with $95 \% \mathrm{O}_{2} / 5 \% \mathrm{CO}_{2}$ and maintained at room temperature $\left(22-24^{\circ} \mathrm{C}\right)$ at $\mathrm{pH}$ 7.6. Suction electrodes were used for stimulation and recording of cranial nerves. The US was a twofold threshold single shock applied to the trigeminal nerve and the CS was a $100 \mathrm{~Hz}, 1 \mathrm{~s}$ train stimulus applied to the ipsilateral auditory nerve that was below threshold amplitude required to produce activity in the abducens nerve. Neural responses were recorded from the ipsilateral abducens nerve that innervates the extraocular muscles controlling movements of the eye, nictitating membrane, and eyelid. In pond turtles, the trigeminal and auditory nerves project directly to the abducens motor neurons (Keifer and Mokin, 2004). The CS-US interval, which was defined as the time between the CS offset and US onset, was $20 \mathrm{~ms}$. Intertrial interval between the paired stimuli was $30 \mathrm{~s}$. A pairing session was composed of 50 CS-US presentations followed by a 30 min rest period during which no stimuli were delivered. Conditioned responses were defined as abducens nerve activity that occurred during the CS and exceeded an amplitude of at least twofold above the baseline recording level. Pseudoconditioning consisted of the same number of CS and US stimuli that were explicitly unpaired using a CS-US interval randomly selected between $300 \mathrm{~ms}$ and $25 \mathrm{~s}$.

Pharmacology. BDNF (Santa Cruz Biotechnology) stock solution was added to physiological saline to a final concentration of $100 \mathrm{ng} / \mathrm{ml}$ and was applied for $80 \mathrm{~min}$, the time duration equivalent to two pairing sessions of conditioning. The selective membrane-permeable cAMP analog and competitive inhibitor of cAMP-dependent protein kinase A (PKA) activation Rp-cAMPs (50 $\mu$; Sigma) or the MEK-ERK inhibitor PD98059 (50 $\mu \mathrm{M}$; Calbiochem) was dissolved in physiological saline and applied to the bath $30 \mathrm{~min}$ before the conditioning procedure or BDNF treatment and continued throughout the experiment. To activate ephrin-B1, preparations were treated with preclustered recombinant human EphB2-Fc ( $5 \mu \mathrm{g} / \mathrm{ml}$; R\&D Systems) for $1.5 \mathrm{~h}$ before conditioning or preclustered human Fc ( $5 \mu \mathrm{g} / \mathrm{ml}$; R\&D Systems) as a control. To activate EphB2, preparations were treated with preclustered recombinant human ephrin-B1-Fc ( $5 \mu \mathrm{g} / \mathrm{ml}$; R\&D Systems). Preclustering was accomplished by incubation of EphB2-Fc, ephrin-B1-Fc, or Fc alone with goat antihuman $\mathrm{Fc}$ antibody (Jackson ImmunoResearch) in a 1:2 ratio on ice for $1 \mathrm{~h}$ (Moeller et al., 2006).

Tracer and antibody injections. Immediately after the end of the conditioning procedures, preparations were injected with anterograde tracer into the auditory nerve and retrograde tracer into the abducens nerve. Anterograde injections were accomplished using a micropipette (tip diameter, $\sim 15 \mu \mathrm{m}$ ) filled with $10 \%$ fluororuby (FR, red; Invitrogen) dissolved in physiological saline. A total volume of $\sim 0.2-0.4 \mu \mathrm{l}$ of FR was injected slowly into the cut end of the auditory nerve over a period of $1 \mathrm{~h}$. In the same preparation, retrograde labeling of abducens motor neurons was achieved by injections of $\sim 0.4 \mu \mathrm{l}$ of $0.5 \%$ Alexa Fluor 488 (AF488, green; Invitrogen) into the ipsilateral abducens nerve for the same time period. Preparations remained in oxygenated physiological saline for an additional $4 \mathrm{~h}$ to allow for transport of tracers for a total elapsed time of $5 \mathrm{~h}$ between the end of training and fixation. In this preparation, these tracers are transported to their targets in 2-3 h (Keifer and Mokin, 2004) and are expected to fully label boutons or somata by the $5 \mathrm{~h}$ time point. Brainstems were then immersion fixed in 3\% paraformaldehyde, cryo- protected, and sectioned at $60 \mu \mathrm{m}$ on a microtome. In some experiments (Li and Keifer, 2008), primary antibodies against BDNF $(200 \mu \mathrm{g} / \mathrm{ml}$; Santa Cruz Biotechnology) or nerve growth factor (NGF; Santa Cruz Biotechnology) were injected into the cut end of the auditory nerve for anterograde transport into presynaptic boutons. The antibodies were delivered at a constant rate using a syringe pump for $6 \mathrm{~h}$. Following these injections, preparations underwent the conditioning procedure, were immersion fixed and processed with Cy3-conjugated secondary antibodies to visualize BDNF or NGF immunoreactivity in boutons. In other experiments, preparations were injected with anti-ephrin-B antibodies ( $5 \mu \mathrm{g} / \mathrm{ml}$; Santa Cruz Biotechnology; selective for ephrin-B1/2/3) into either the auditory (to fill presynaptic boutons) or abducens (to fill postsynaptic abducens motor neurons) nerves using a syringe pump for a $5 \mathrm{~h}$ transport time before the physiological experiments.

Imaging and morphological analysis. Images were captured using a Zeiss Axioskop Mot 2 microscope equipped with an Axiocam digital color camera. A $63 \times$ NA 1.4 oil-immersion objective and dual excitation/emission cube were used to image AF488 retrogradely labeled abducens motor neurons and FR anterogradely labeled auditory nerve boutons. Images were analyzed using Image (NIH). Only boutons observed to be directly overlapping with a portion of a retrogradely labeled neuron and in the same focal plane were determined to be in apposition to abducens motor neurons and included in the analysis. The following parameters were measured: bouton diameter, defined as the maximum width of a bouton, and bouton area. Data from boutons observed on proximal and distal dendrites were collapsed as there were no differences between the groups. Data were analyzed using a one-way ANOVA followed by post hoc analysis using Fisher's and Bonferroni's tests. Values are presented as means \pm SEM.

Subcellular fractionation and Western blot. Subcellular fractions were prepared according to Zhou et al. (2007) with some modification. All procedures were performed at $4^{\circ} \mathrm{C}$ in the presence of protease and phosphatase inhibitors. Brainstem samples were obtained by dissecting a thick slab ( $\sim 2 \mathrm{~mm}$ ) of tissue from the pons containing the abducens nuclei. To obtain enough tissue, three brainstem samples were pooled for each group and homogenized in ice-cold sucrose/HEPES buffer $(0.32 \mathrm{~m} \mathrm{su}-$ crose and $10 \mathrm{~mm}$ HEPES). The homogenates were centrifuged and the synaptosome layer was resuspended in sucrose/HEPES buffer and ultracentrifuged at $160,000 \times g$ for $15 \mathrm{~min}$. Purified synaptosomes were diluted and centrifuged at $40,000 \times g$ for $30 \mathrm{~min}$. The pellets were resuspended, centrifuged at $40,000 \times g$ to yield the purified PSD fraction. Presynaptic fractions were recovered using rapid $\mathrm{pH}$ changes in solubilization buffer and centrifugation at 18,000 $\times g$ for $30 \mathrm{~min}$. For Western blotting, equal amounts of protein were subjected to SDS-PAGE. Blots were probed with anti-synaptophysin (1:1000; Sigma), anti-PSD-95 (1: 1000; Cell Signaling Technology), anti-GluR1 (1:1000; Millipore), antiephrin-B1 (1:500), and anti-EphB2 (1:2000; R\&D Systems). Proteins were detected using chemiluminescence and immunoreactive signals were captured on Kodak X-omatic AR film and quantified by computerassisted densitometry. Membranes were reprobed for loading controls with an anti- $\beta$-actin antibody (1:1000; Millipore).

Immunoprecipitation. For determination of phosphorylation state of tyrosine and serine residues of EphB2 and ephrin-B1, physiological experiments were performed and brainstems immediately frozen in liquid nitrogen. Protein samples were precleared with Protein G-Agarose and incubated with anti-ephrin-B1 or anti-EphB2 antibodies (R\&D Systems) complexed with Protein G-Agarose overnight at $4^{\circ} \mathrm{C}$. Immunoprecipitates were washed with ice-cold lysis buffer containing $500 \mathrm{~mm} \mathrm{NaCl}$ and dissociated by heating for $3 \mathrm{~min}$ in the loading buffer. Proteins were detected by Western blot using anti-phosphotyrosine (1:1000; Invitrogen) or anti-phosphoserine (1:1000; Invitrogen) primary antibodies.

\section{Results}

\section{Selective growth of presynaptic auditory boutons after conditioning}

To assess whether there were presynaptic structural modifications after conditioning, tract tracing was used to visualize auditory nerve presynaptic boutons anterogradely labeled with FR 

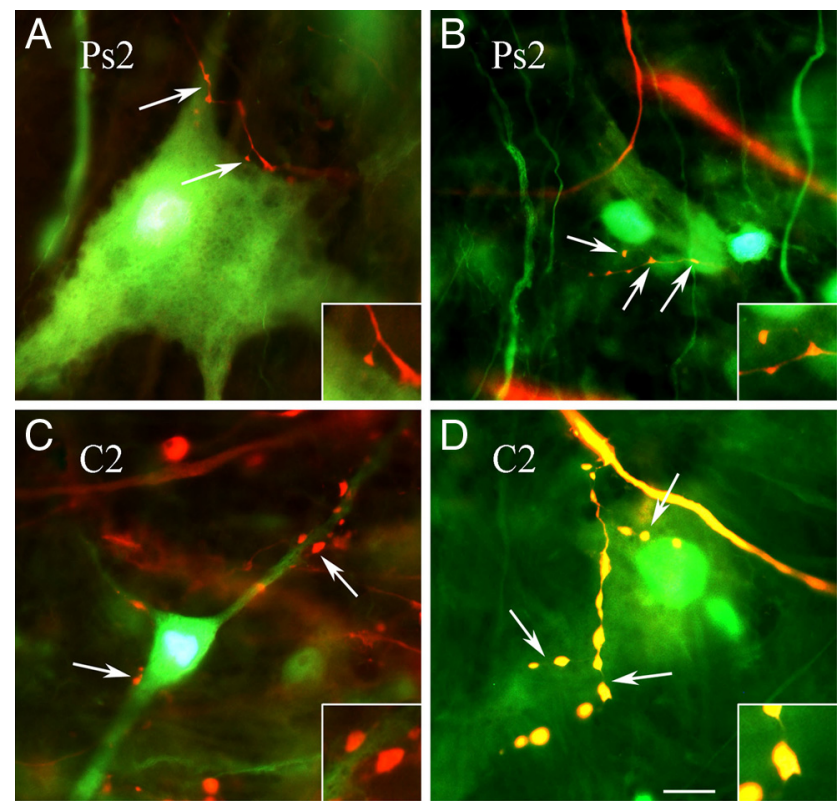

Figure 1. Photomicrographs of anterogradely labeled auditory nerve presynaptic boutons with fluororuby (red) apposed to retrogradely filled abducens motor neurons with Alexa Fluor 488 (green) from pseudoconditioned ( $\boldsymbol{A}, \boldsymbol{B} ; \mathrm{Ps} 2)$ and conditioned (C, $;$; 2 ) preparations. Arrows indicate boutons in apposition to motor neurons. Insets show higher magnification of boutons. Scale bar, $10 \mu \mathrm{m}$.

(red) apposed to postsynaptic abducens motor neurons retrogradely labeled with AF488 (green) (Keifer and Mokin, 2004). Following the conditioning procedure and acquisition of abducens nerve CRs (to a mean of $66 \pm 9 \%$ compared with $0 \%$ CRs after pseudoconditioning; $p<0.0001, n=6$ preparations/group) tracer substances were injected into the nerves and allowed to transport for a total elapsed time of $5 \mathrm{~h}$ between the end of training and fixation. Photomicrographs of auditory nerve boutons in apposition to abducens motor neurons from preparations that were either pseudoconditioned (Fig. $1 A, B$ ) (Ps2) or conditioned (Fig. 1C,D) (C2) for two pairing sessions are shown. Boutons (arrows) from conditioned preparations appear larger than those from the pseudoconditioned group. In Figure $1 A$, an axon originating from the auditory nerve is observed to have two small en passant boutons apposed to the proximal dendrite of an abducens motor neuron (upper arrow) and one bouton in contact with the soma (lower arrow) as a terminaux contact. Small en passant boutons apposed to a motor neuron soma are also shown in Figure $1 B$. The average area of the boutons shown in Figure $1 A$ is $1.20 \mu \mathrm{m}$, while that of those in Figure $1 B$ is $0.76 \mu \mathrm{m}$. In contrast, images taken from the conditioned group show several large boutons apposed to proximal (lower left arrow) and distal (upper right arrows) dendrites of one neuron (Fig. 1C) and a number of large boutons on the proximal dendrite and soma of another (Fig. $1 D)$. The average area of these boutons is $1.52 \mu \mathrm{m}$ and $3.05 \mu \mathrm{m}$, respectively. Quantitative analysis showed that the average diameter of all auditory nerve boutons that were apposed to the dendrites or soma of abducens motor neurons was significantly greater after conditioning, having a mean of $1.57 \pm 0.03 \mu \mathrm{m}$ (Fig. $2 A$ ) (total boutons, $n=515$ boutons) compared with pseudoconditioning at $1.37 \pm 0.03 \mu \mathrm{m}(n=321, p<0.0001)$. Similarly, the average area of boutons was $1.31 \pm 0.07 \mu \mathrm{m}(n=321)$ after pseudoconditioning compared with $1.59 \pm 0.05 \mu \mathrm{m}(n=515)$ after conditioning (Fig. $2 C$ ) (total boutons, $p=0.001$ ). Cumulative distribution plots verify the significant rightward shift to- ward larger bouton area after conditioning compared with pseudoconditioning $(p<0.0001)$ (Fig. $2 E$ ). To examine whether the enhanced size of auditory boutons after conditioning was related to their morphological localization on abducens motor neurons, the sample of all boutons was divided into those apposed to somata or apposed to dendrites. Strikingly, both the diameter and area of boutons apposed to the dendrites were found to be significantly greater after conditioning while those apposed to somata were not (Fig. 2A,C) (dendritic boutons, $p<$ 0.0001 for both diameter and area compared with Ps2). To examine whether the observed changes in size selectively involved boutons directly apposed to abducens motor neurons, nearby boutons not in contact with abducens neurons were measured. This set of boutons was unequivocally attached to the same axon as those that were in apposition. Analysis showed that conditioning did not result in significant changes in either the diameter or area of nonapposed boutons compared with pseudoconditioning (Fig. $2 B, D)(n=294, p=0.63$ diameter, $p=0.74$ area). Representative photomicrographs (Fig. $2 G-I$ ) illustrate these changes in bouton size for those in apposition (arrows) or not in apposition (arrowheads) to abducens neurons. Together, these data indicate that auditory nerve boutons selectively localized to the dendrites but not the somata of abducens motor neurons undergo significant growth after conditioning. Importantly, this effect was observed only for boutons in apposition to dendritic processes and not for those located in close proximity.

\section{BDNF is required for bouton growth during conditioning}

Previously, we reported that exogenously applied BDNF induced postsynaptic trafficking of AMPARs and enhanced expression of the presynaptic proteins synaptophysin and synapsin I in this preparation (Li and Keifer, 2008). Here, we asked whether BDNF is also involved in the conditioning-induced presynaptic morphological changes observed here. Bath application of BDNF for $80 \mathrm{~min}$, the equivalent time period of two pairing sessions, resulted in a significant increase in bouton size compared with pseudoconditioning (Fig. $2 A, C)(n=3$ preparations; total boutons, $n=236, p=0.0006$ diameter, $p=0.0008$ area, BDNF2 vs Ps2). This finding is also illustrated in the cumulative distribution plot of bouton area $(p<0.0001)$ (Fig. $2 F)$. Interestingly, when boutons were divided into those apposed to dendrites or somata, a significant increase in bouton size was found only for those apposed to the dendrites (Fig. 2A,C) (dendritic boutons, $n=$ 123 , diameter and area, $p<0.0001$, BDNF2 vs Ps2). However, unlike conditioning, BDNF treatment also resulted in significantly increased size of nearby nonapposed boutons (Fig. $2 B, D$ ) $(n=151, p=0.03$ diameter, $p=0.02$ area, BDNF2 vs Ps2) as illustrated in Figure $2 \mathrm{~J}$. In an earlier study (Li and Keifer, 2008), anti-BDNF or NGF antibodies were injected into the auditory nerve that conveys the CS for anterograde transport before conditioning. Conditioning failed to be acquired in the BDNF antibody-treated preparations while normal levels of CRs were obtained in preparations injected with NGF antibodies. To determine whether BDNF is required for bouton enlargement, we reexamined the imaging data obtained in that study. Quantitative analysis indicated that boutons treated with BDNF antibodies were indistinguishable in size from those that received pseudoconditioning (Fig. 3) ( $n=6$ preparations/group, $p=0.22$ diameter, $p=0.88$ area, $\mathrm{BDNF} \mathrm{Ab}$ vs Ps2). By comparison, preparations injected with NGF antibodies showed significantly enlarged boutons after conditioning similar to conditioning in untreated preparations (Fig. 3) $(p=0.006$ diameter, $p<0.0001$ area, NGF Ab vs BDNF Ab). These results suggest a critical role 

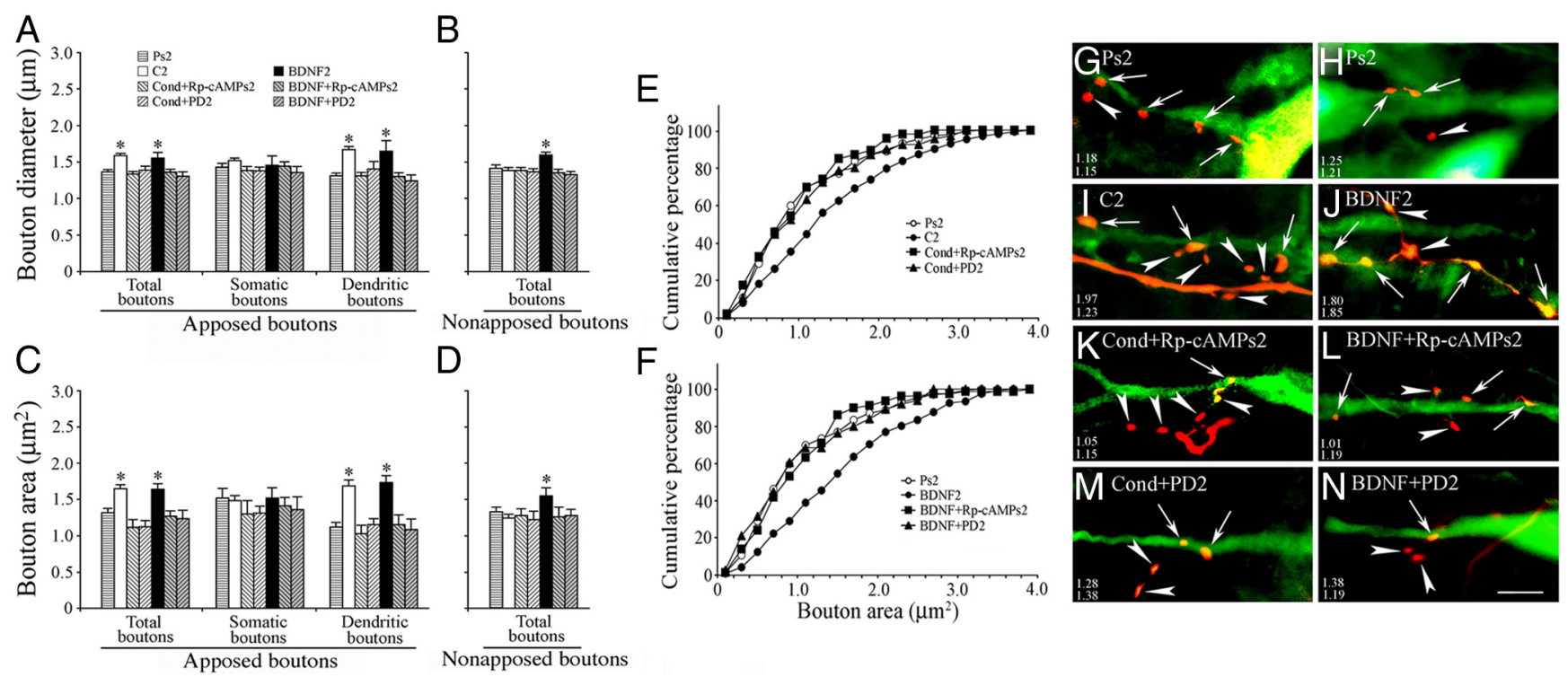

Figure 2. Conditioning and BDNF treatment promote selective auditory nerve bouton growth on abducens neuron dendrites but not somata. $A, C$, Conditioning and BDNF treatment resulted in a significant increase in bouton diameter $(A)$ and area $(C)$ that was blocked by bath application of Rp-cAMPs or PD98059. The increase in size of the total number of boutons stemmed from boutons apposed to the dendrites but not the somata of abducens motor neurons. $\boldsymbol{B}, \boldsymbol{D}$, Nearby boutons that were not in direct apposition to motor neurons did not exhibit changes in diameter $(\boldsymbol{B})$ and area (D) after conditioning, while they did show enlargement following BDNF treatment. The BDNF-induced enhancement in size of nonapposed boutons was abolished by coapplication with Rp-cAMPs or PD98059. *Significant difference $(p<0.05)$ from Ps2 or BDNF2. $p$ values are given in the text. $\boldsymbol{E}, \boldsymbol{F}$, Cumulative distribution plots of total bouton area for the different experimental groups. There is a significant rightward shift toward larger boutons after conditioning $(\boldsymbol{E})$ and BDNF-treatment $(\boldsymbol{F})$ compared with the other treatment groups. $\mathbf{G}-\boldsymbol{N}$, Photomicrographs of representative auditory nerve boutons apposed (arrows) or not apposed (arrowheads) to abducens neurons from the different experimental groups. The average diameter of the boutons shown apposed (upper numbers) or not apposed (lower numbers) to motor neurons in each panel is indicated. Scale bar, $5 \mu \mathrm{m}$.

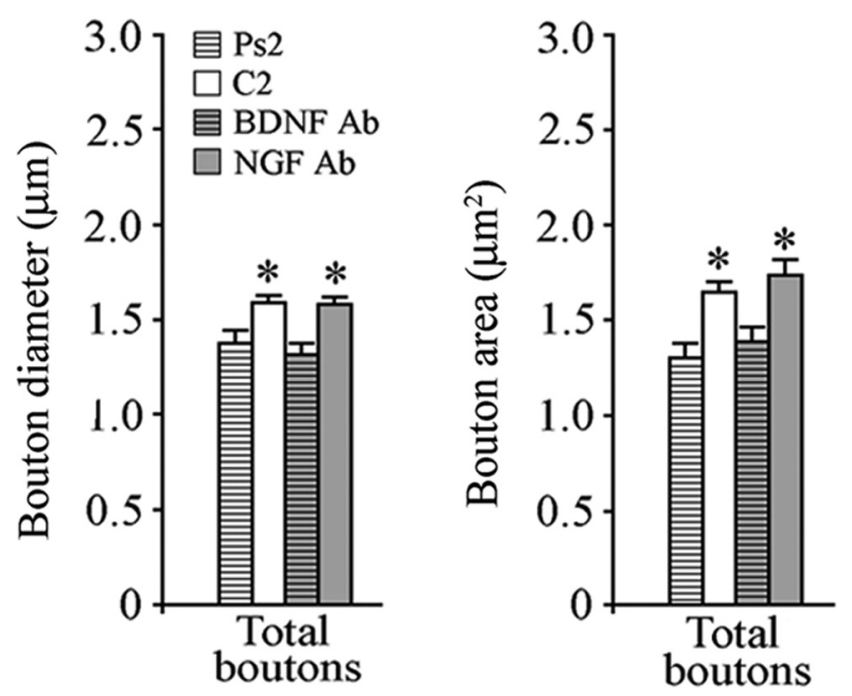

Figure 3. Presynaptic BDNF is required for bouton enlargement. The diameter and area of boutons from preparations subjected to presynaptic auditory nerve injections of BDNF antibodies (Ab) before conditioning was similar to that from pseudoconditioned preparations. In contrast, presynaptic injections of NGF antibodies failed to inhibit conditioning-induced bouton enlargement.

for presynaptic BDNF, but not NGF, in auditory nerve bouton growth during conditioning.

\section{PKA and ERK are required for bouton growth}

Signaling cascades involving PKA and ERK have been shown to have a critical role in in vitro classical conditioning (Zheng and Keifer, 2009). To examine whether PKA and ERK play a role in presynaptic bouton enlargement during conditioning, the PKA inhibitor Rp-cAMPs or the MEK-ERK inhibitor PD98059 was applied to the bath before training. The electrophysiological results showed that few CRs were expressed during treatment; $0 \%$ CRs during Rp-cAMPs application and $5 \pm 9 \%$ in the presence of PD98059. Preparations that underwent the conditioning procedure during treatment in the presence of Rp-cAMPs ( $n=3$ preparations) or PD98059 $(n=3)$ showed bouton sizes equivalent to control values for the total sample as well as for boutons localized to the soma or dendrites (Fig. $2 A, C$ ) (total boutons, $n=73$ boutons, $p=0.68$ diameter, $p=0.15$ area, Rp-cAMPs2 vs Ps2; $n=131, p=0.68$ and $p=0.42$, PD2 vs Ps2). This finding is also illustrated in the cumulative distribution plot of total bouton area (Fig. 2E). Moreover, the size of nearby nonapposed boutons was also similar to control (Fig. $2 B, D)(n=91, p=0.60$ diameter, $p=0.56$ area, Rp-cAMPs2 vs Ps $2 ; n=70, p=0.85$ diameter, $p=$ 0.97 area, $\mathrm{PD} 2$ vs Ps2). Photomicrographs showing boutons from preparations treated with Rp-cAMPs or PD98059 before conditioning are shown in Figure 2, $K$ and $M$. The effect of Rp-cAMPs ( $n=3$ preparations) and $\operatorname{PD98059}(n=3)$ on bouton size of preparations treated with BDNF was also investigated. Similar to the results during conditioning, both compounds prevented the enhancement in bouton size induced by BDNF treatment (Fig. $2 A, C, F)$ (total boutons, $n=148, p=0.004$ diameter, $p=0.002$ area, Rp-cAMPs2 vs BDNF2; $n=82, p=0.001$ diameter, $p=$ 0.005 area, $\mathrm{PD} 2$ vs BDNF2). These findings are illustrated in Figure 2, $L$ and $N$. Interestingly, these compounds also inhibited the BDNF-induced enlargement of nearby nonapposed boutons (Fig. $2 B, D)(n=87, p=0.008$ diameter, $p=0.04$ area, Rp-cAMPs2 vs BDNF2; $n=108, p=0.001$ diameter, $p=$ 0.04 area, $\mathrm{PD} 2$ vs BDNF2).

\section{Synaptic localization of EphB2 and ephrin-B1}

Presynaptic or postsynaptic localization of EphB/ephrin-B is key to their roles in synaptic plasticity and learning. To examine the function of EphB2 and ephrin-B1 in in vitro classical condition- 


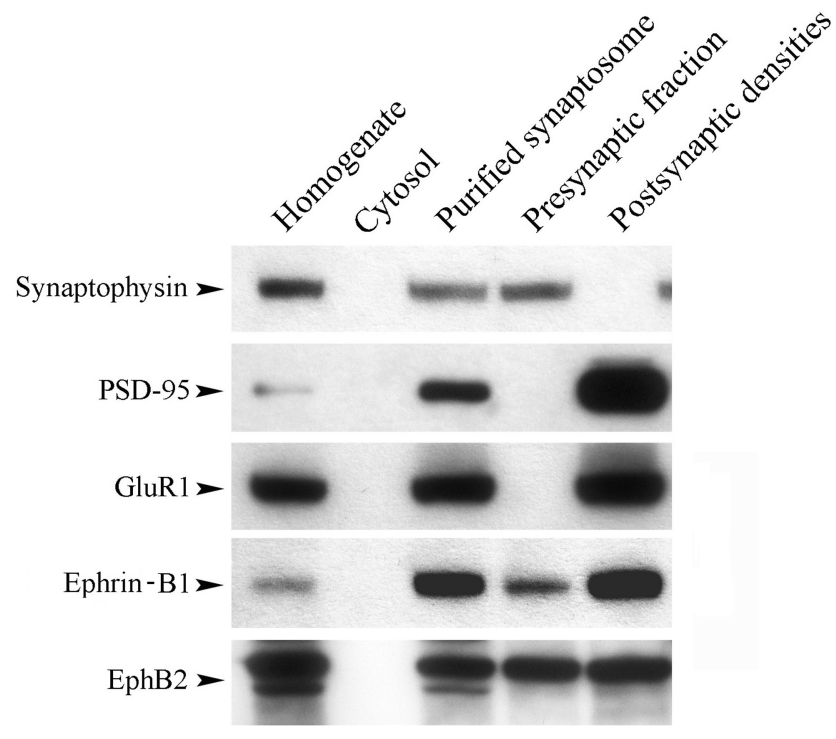

Figure 4. Ephrin-B1 is localized mainly presynaptically, while EphB2 is present both presynaptically and postsynaptically in subcellular fractions of tissue samples containing the abducens motor nuclei. The presynaptic vesicle-associated protein synaptophysin was found in the presynaptic fraction, whereas the PSD fraction contained representative postsynaptic proteins such as PSD-95 and AMPAR subunit GluR1. The subcellular distribution of ephrin-B1 was mainly postsynaptic, while EphB2 was localized both presynaptically and postsynaptically in relatively equal amounts. Western blot taken from a naive unstimulated preparation. Protein distribution was not altered by pseudoconditioning or conditioning.

ing, differential centrifugation followed by Western blot analysis was performed to determine their subcellular distribution (Fig. 4). Tissue from naive (unstimulated) preparations containing the abducens motor nuclei showed that the presynaptic fraction contained the synaptic vesicle-associated protein synaptophysin while the PSD fraction was enriched with characteristic proteins such as PSD-95 and the AMPAR subunit GluR1. All of these proteins were detected in the purified synaptosome that contained both presynaptic and postsynaptic membranes and were absent in the cytosolic fraction, further confirming that the fractions were uncontaminated. This analysis showed that ephrin-B1 was mainly localized to the postsynaptic fraction while EphB2 was present on both sides of the synapse in relatively equal amounts. Similar analysis of pseudoconditioned or conditioned tissue had no detectable effect on the subcellular distribution of EphB2 or ephrin-B1 protein.

\section{Postsynaptic activation of ephrin-B1 during BDNF signaling} and conditioning

The phosphorylation state of ephrin-B1 and EphB2 was determined after conditioning and BDNF application. Immunoprecipitation with anti-ephrin-B1 or anti-EphB2 antibodies followed by immunoblotting with anti-phosphotyrosine or anti-phosphoserine antibodies was performed. Conditioning resulted in a significant increase in phosphorylation levels of both tyrosine ( $n=5$ /group, $p=0.001)$ and serine $(p<0.0001)$ residues of ephrin-B1 compared with pseudoconditioning (data not shown). In contrast, no changes were found in levels of phosphotyrosine $(p=0.51)$ and phosphoserine $(p=0.41)$ of EphB2 after conditioning. Similarly, application of BDNF also induced increased levels of phosphotyrosine $(p<0.0001)$ and phosphoserine $(p=0.003)$ residues of ephrin-B1 compared with untreated preparations but had no effect on EphB2 ( $p=0.94$, phosphotyrosine; $p=0.62$, phospho- serine). Therefore, both conditioning and BDNF application result in phosphorylation of ephrin-B1 but not EphB2.

To further assess whether conditioning is associated with activation of postsynaptically localized ephrin-B1, we used preclustered EphB2-Fc, a chimera containing the extracellular domain of EphB2 fused to the Fc portion of human IgG, to activate ephrin-B1 (Moeller et al., 2006; Lim et al., 2008). Preparations treated with EphB2-Fc exhibited significantly increased levels of phosphotyrosine (Fig. 5A,B) $(n=4$ /group, $p<0.0001)$ and phosphoserine $(p<0.0001)$ of ephrin-B1 compared with preparations treated with $\mathrm{Fc}$ alone. To inactivate postsynaptic ephrin-B, antibodies raised against the intracellular domain of ephrin-B were injected into the abducens nerve and resulted in suppressed EphB2-Fc-induced phosphorylation of ephrin-B1 to near baseline levels (Fig. $5 A, B)[p=0.09$, phosphotyrosine; $p=$ 0.10, phosphoserine, EphB2-Fc+Ab(post) vs Fc]. Significantly, preparations injected postsynaptically with the same antibodies during the conditioning procedure also showed inhibition of ephrin-B1 phosphorylation (Fig. $5 A, B)[p=0.07$, phosphotyrosine; $p=0.49$, phosphoserine, $\mathrm{C} 2+\mathrm{Ab}$ (post) vs $\mathrm{Fc} ; 2 \pm 0.8 \%$ CRs], while those subjected to presynaptic auditory nerve injection exhibited increased levels of ephrin-B1 phosphorylation that were comparable to normal conditioning $[p<0.0001$, phosphotyrosine; $p=0.0005$, phosphoserine, $\mathrm{C} 2+\mathrm{Ab}$ (pre) vs Fc; $50 \pm 9 \% \mathrm{CRs}$. As with conditioning, BDNF signaling was also dependent on postsynaptic ephrin-B1 activation. Preparations injected presynaptically with ephrin-B antibodies before BDNF bath application showed a significantly high level of phosphorylation of ephrin-B1 that was comparable to results with BDNF alone (Fig. $5 A, B)[p<0.0001$, phosphotyrosine; $p=0.001$, phosphoserine, $\mathrm{BDNF} 2+\mathrm{Ab}$ (pre) vs Fc]. However, inhibition of phosphorylation was observed in preparations that received postsynaptic injections $[p=0.34$, phosphotyrosine; $p=0.23$, phosphoserine, BDNF2 $+\mathrm{Ab}$ (post) vs Fc] Therefore, these data indicate that postsynaptic activation of ephrin-B1 is associated with both BDNF signaling and classical conditioning.

\section{Presynaptic EphB2 mediates auditory bouton growth}

Since postsynaptic ephrin-B1 is activated during conditioning and BDNF application, we examined whether EphB2 is also involved in presynaptic auditory nerve bouton growth. As observed earlier, conditioned preparations showed significantly greater diameter and area of the total population of boutons compared with the pseudoconditioned group (Fig. $6 A, C)(n=6$ preparations, 515 and 312 boutons, respectively; $p<0.0001$ diameter, $p=0.001$ area, C2 vs Ps2). Similarly, further analysis with respect to morphological localization on abducens motor neurons showed that the diameter and area of boutons apposed to dendrites were significantly greater $(p<0.0001$ diameter and area vs Ps2), while those of boutons apposed to somata were not ( $p=$ 0.15 diameter; $p=0.77$ area). In addition, the size of nearby boutons not apposed to abducens neurons also remained unchanged by conditioning (Fig. $6 B, D)(p=0.63$ diameter; $p=$ 0.74 area vs Ps2). Significantly, in this set of experiments, there was no increase in bouton diameter or area in preparations subjected to postsynaptic injection of ephrin-B antibodies followed by conditioning [total boutons, $n=127$ boutons/ 3 preparations; $p=0.95$ diameter, $p=0.91$ area; dendritic boutons, $p=0.43$ diameter, $p=0.99$ area, $\mathrm{C} 2+\mathrm{Ab}$ (post) vs Ps2; mean of $0 \% \mathrm{CRs}$ ]. Representative images showing boutons from these groups are illustrated in Figure $6 E-G$. Similar to conditioning, BDNF treatment also resulted in a significant increase in bouton size compared with pseudoconditioning, as expected (Fig. 6A,C) (236 
boutons/3 preparations; $p=0.0006 \mathrm{di}-$ ameter; $p=0.0008$ area, BDNF2 vs Ps2). Analysis by morphological localization showed a significant increase in bouton size only for those apposed to the dendrites $(p<0.0001$, diameter and area, BDNF2 vs Ps2), and nearby nonapposed boutons also showed an increase in size (Fig. $6 B, D)(p=0.03$ diameter, $p=0.02$ area, BDNF2 vs Ps2). Again, the BDNF-induced enlargement of dendritic boutons was blocked by postsynaptic injection of ephrin-B antibodies [total boutons, $n=142$ boutons/3 preparations, $p=0.0025$ diameter, $p<0.0001$ area; dendritic boutons, $p=0.0085$ diameter, $p=0.0001$ area, $\mathrm{BDNF} 2+\mathrm{Ab}$ (post) vs BDNF2], but the size of nonapposed boutons was unaffected (Fig. $6 B, D)(p=0.79$ diameter; $p=0.72$ area). Given our findings above, the effects of postsynaptic ephrin-B on presynaptic bouton size were likely to be mediated by presynaptically localized EphB2. Therefore, we reasoned that treatment with ephrin-B1-Fc should rescue the inhibitory effect of the ephrin- $\mathrm{B}$ antibody on bouton size. To confirm that ephrin-B1-Fc acted as an EphB2 activator, we examined phosphorylation levels of Eph/ephrin in treated preparations. As shown in Figure $6 K$, ephrin-B1-Fc treatment resulted in no change in phosphorylation of ephrin-B1 compared with $\mathrm{Fc}$ alone, while the same treatment dramatically increased the phosphorylation state of EphB2. Similar findings were also reported by Lim et al. (2008) using Xenopus tadpole brain. Moreover, using subcellular fractionation, EphB2 localized to the presynaptic fraction was more heavily phosphorylated during ephrin-B1-Fc application compared with the postsynaptic fraction (Fig. $6 \mathrm{~K}$ ). As predicted, the average dendritic bouton diameter was significantly increased by bath application of ephrin-B1-Fc to levels similar to those observed after BDNF treatment alone and rescued the inhibitory effects of postsynaptically injected ephrin-B antibodies [total boutons, $n=207$ boutons $/ 3$ preparations, $p=0.006$ diameter; $p=0.001$ area; dendritic boutons, $p=0.001$ diameter; $p<0.0001$ area, $\mathrm{BDNF} 2+\mathrm{Ab}$ (post) + ephrin-B1-Fc vs Ps2]. Representative images of these changes in bouton size are shown in Figure $6 \mathrm{H}-\mathrm{J}$. These results suggest that postsynaptic ephrin-B forward signaling to EphB2 functions to modify the size of presynaptic boutons.

\section{Discussion}

\section{Selective bouton growth on dendritic processes} during conditioning

Functional changes in synaptic strength are often accompanied by observable remodeling of synaptic morphology (Holtmaat and Svoboda, 2009). In the present study, we found that auditory nerve presynaptic boutons that convey the CS to abducens motor neurons during classical conditioning undergo selective growth. Conditioning-induced enlargement occurred for boutons in direct apposition to motor neurons rather than for boutons located nearby but not in apposition. This strongly suggests that bouton enlargement is not due to nonspecific effects such as electrical stimulation that might trigger synaptic swelling. The underlying mechanisms that induce presynaptic bouton growth are poorly understood. Here, we provide evidence that BDNF plays a pivotal role in this process. Bath application of BDNF alone to prepara- tions promotes increased bouton size to a similar degree as during conditioning. In support of this finding, injections of antiBDNF antibodies into the auditory nerve prevented bouton enlargement as well as CR acquisition while anti-NGF antibodies similarly injected failed to suppress bouton growth and acquisition. Significantly, only those boutons contacting abducens motor neuron dendrites rather than somata increased in size after conditioning. The question arises as to how this selective enlargement is achieved, since boutons apposed to both the soma and dendrites of abducens neurons receive the same conditioning stimuli. An intriguing recent study using cultured neurons found that BDNF transcripts containing short $3^{\prime}$ untranslated regions (UTRs) are restricted to the soma while those with long UTRs are targeted to the dendrites (An et al., 2008). Neurons deficient in long 3' UTR BDNF mRNAs display impairment in regulated BDNF secretion, a loss of hippocampal LTP, and decreased dendritic spine size. These authors suggested that somatic BDNF encoded from the short 3' UTR mRNAs is responsible for neuronal survival whereas dendritic BDNF from long UTRs is involved in functional and structural plasticity. Although this hypothesis on the subcellular regulation of BDNF function by UTRs is derived from work on postsynaptic spines, BDNF is also likely to participate in presynaptic bouton remodeling as a secreted molecule. One possibility is that the failure of somatic bouton growth during conditioning is due to the lack of local synaptic BDNF release and that, conversely, abundantly secreted $\mathrm{BDNF}$ in the region of the dendrites results in increased size of dendritic boutons. Extending this idea further, it has been shown that plasticity-related BDNF availability can also be controlled by extracellular proteases such as plasmin (Pang et al., 2004) and metalloproteinases (Keifer et al., 2009). The mature and active form of BDNF is synthesized as a precursor protein proBDNF that is secreted into the synaptic cleft and subjected to proteolytic cleavage to generate BDNF (Lee et al., 2001; Yang et al., 2009). BDNF is essential for LTP while proBDNF appears to facilitate LTD (Lu et al., 2005). Considering that LTP and LTD are accompanied by an increase and decrease in synaptic size, respectively (Zhou et al., 2004; Yang et al., 2008), it seems plausible that the ratio of proBDNF/BDNF controlled in an activity-dependent manner by extracellular proteases could also determine local syn- 
A
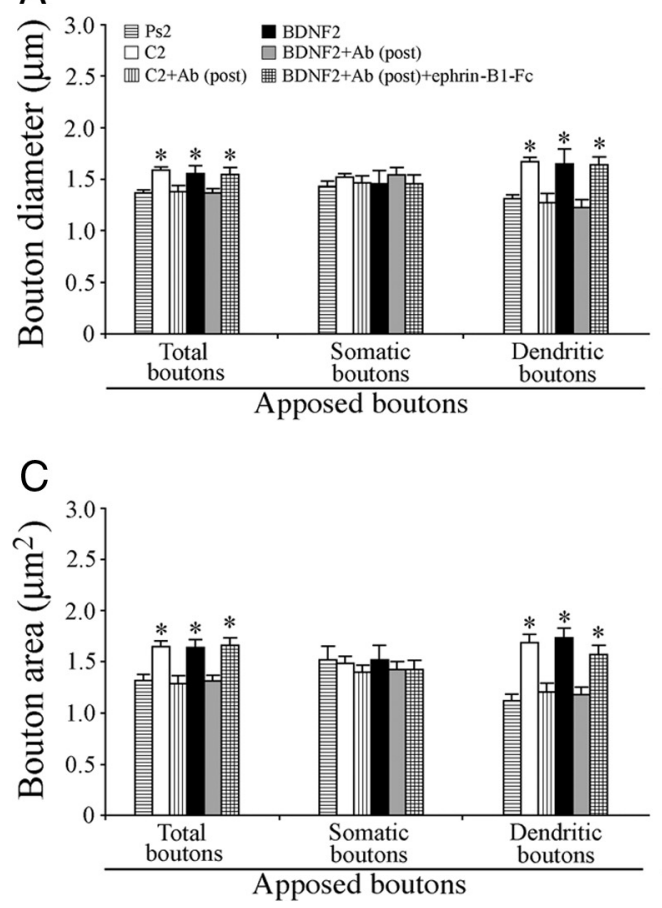

B

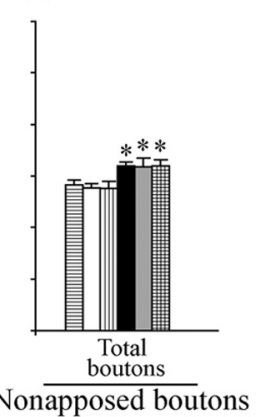

D

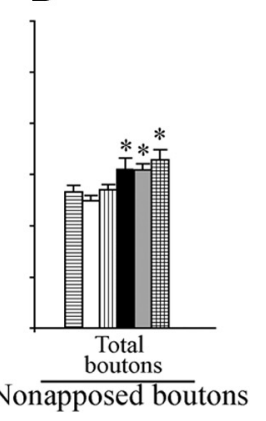

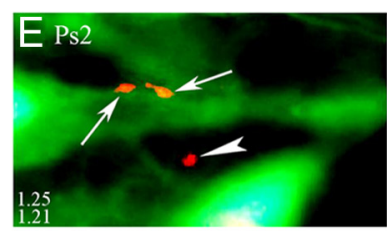
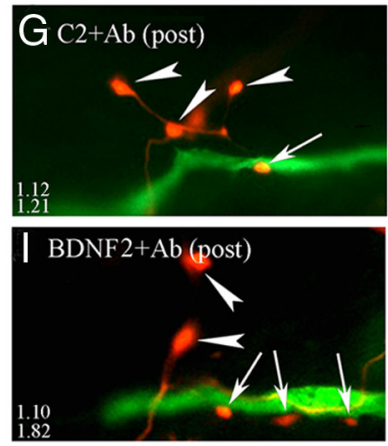

$\mathrm{K}$

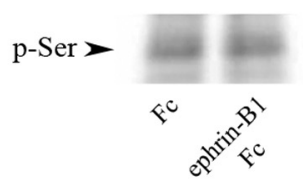

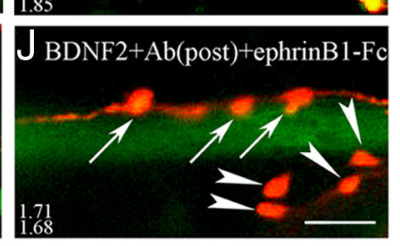
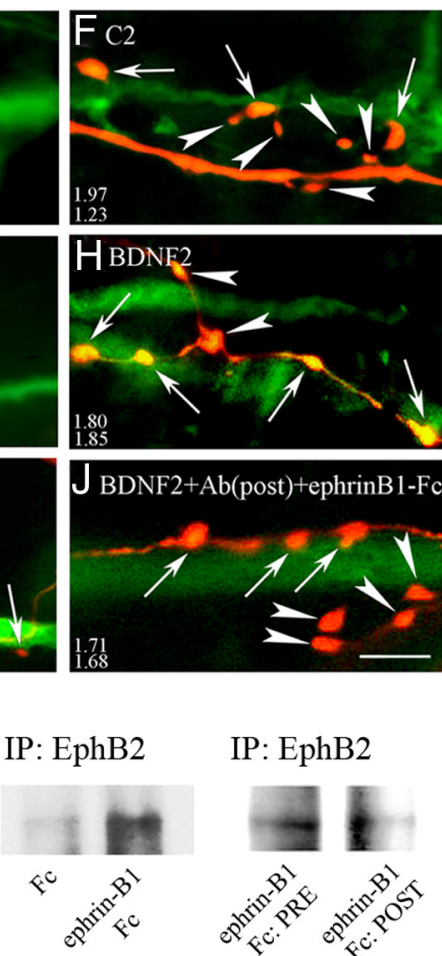

IP: EphB2

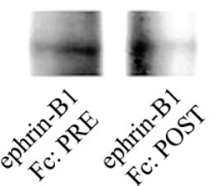

Figure 6. Activation of presynaptic EphB2 underlies growth of presynaptic auditory boutons. $A, C$, Conditioning and BDNF treatment resulted in a significant increase in bouton diameter $(\boldsymbol{A})$ and area $(\boldsymbol{C})$ that was blocked by postsynaptic injections of antibodies directed against ephrin-B $[C 2+A b($ post) and BDNF2 $+A b$ (post) groups]. The increase in total bouton size was from those apposed to the dendrites rather than the somata. Treatment with the EphB2 activator ephrin-B1-Fc during BDNF application and after postsynaptic injection of ephrinB antibodies rescued the effect of the antibody and resulted in enlargement of boutons $[B D N F 2+A b$ (post) + ephrin-B1-Fc].B, $\boldsymbol{D}$, Nearby boutons not apposed to abducens neurons did not exhibit changes in diameter $(\boldsymbol{B})$ and area $(\boldsymbol{D})$ after conditioning, while there was enlargement of boutons following BDNF treatment. The BDNF-induced enhancement in the size of nonapposed boutons was not suppressed by postsynaptic injections of ephrin-B antibodies, and there was no further increase in bouton size after treatment with ephrin-B1-Fc. *Significant difference from Ps2 or BDNF2. E-J, Photomicrographs of representative boutons apposed (arrows) or not apposed (arrowheads) to abducens neurons from the different groups. The average diameter of the boutons shown apposed (upper numbers) or not apposed (lower numbers) to motor neurons is indicated in each panel. Scale bar, $5 \mu \mathrm{m}$. $\boldsymbol{K}$, Ephrin-B1-Fc treatment resulted in no change in levels of ephrin-B1 phosphoserine compared with Fc alone (left), while the same treatment resulted in an increased phosphorylation state of EphB2 (middle). Subcellular fractionation and immunoprecipitation for EphB2 showed greater levels of phosphoserine in presynaptic fractions than in postsynaptic fractions after treatment with ephrin-B1-Fc (right).

apse size and strength. Additionally, the localization of the BDNF receptor tropomyosin-related kinase B (TrkB) may also be involved in selective bouton growth. It remains to be determined whether TrkB is more prevalent at dendritic abducens neuron synapses compared with somatic ones. Such differential targeting is possible since, like BDNF mRNAs, TrkB receptor mRNAs also contain short and long $3^{\prime}$ UTRs (An et al., 2008).

\section{Role for EphB/ephrin-B forward signaling in presynaptic structural plasticity during conditioning}

Here, we provide evidence for ephrin-B-induced EphB2 forward signaling in presynaptic structural plasticity during classical conditioning. We show that inhibition of postsynaptic ephrin-B function by localized antibody injection inhibits ephrin-B1 phosphorylation, presynaptic bouton enlargement, and CR acquisition, and that antibody injections into presynaptic sites fail to show these effects. The suppression of bouton growth is rescued by introduction of the EphB2 activator ephrin-B1-Fc (Lim et al., 2008), which in our preparation activates EphB2 localized to the presynaptic fraction to a greater degree than the postsynaptic fraction. Moreover, the ephrin- $\mathrm{B}$ antibody injections have no effect on the BDNF-induced enlargement of boutons not in apposition to abducens neurons, suggesting that the interaction of presynaptic EphB2 with postsynaptic ephrin-B is essential for bouton growth during conditioning. Together, our data suggest that Eph/ephrin interactions during conditioning specifically function through EphB2 to modify conditioning-related presyn-

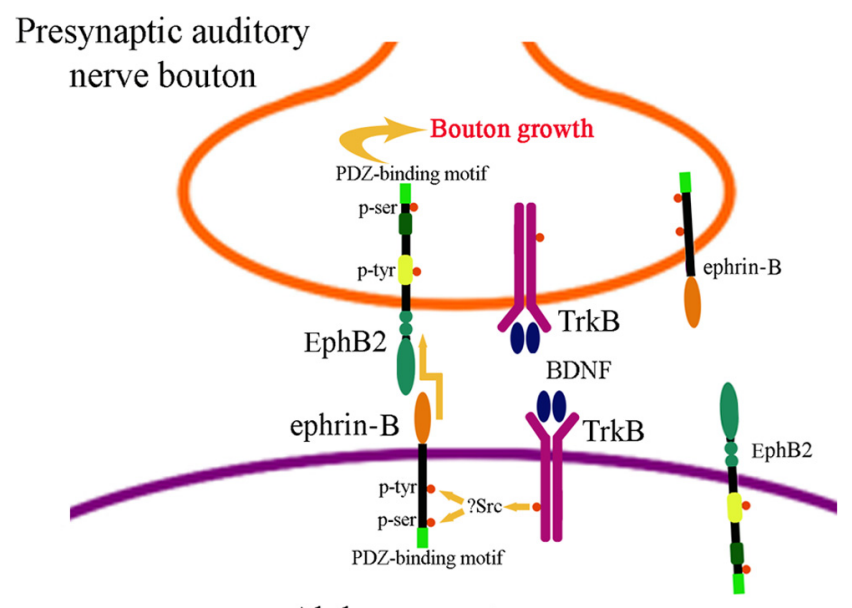

Abducens motor neuron

Figure 7. Schematic diagram illustrating transsynaptic EphB2/ephrin-B forward signaling during conditioning-induced bouton growth. Activation of TrkB receptors by BDNF results in phosphorylation of tyrosine and serine residues of postsynaptic ephrin-B. Forward EphB2 signaling is induced by ephrin-B and results in presynaptic auditory nerve bouton growth through a mechanism that may be independent of EphB2 phosphorylation and instead involves protein-protein interactions of the EphB2 PDZ-binding motif.

aptic morphology. Therefore, we propose that conditioning or BDNF treatment results in presynaptic bouton growth through transsynaptic EphB2/ephrin-B signaling as presented in Figure 7. Briefly, BDNF released extracellularly during conditioning from 
presynaptic and/or postsynaptic compartments, or applied exogenously, binds and activates postsynaptic TrkB and induces phosphorylation of tyrosine and serine residues of ephrin-B probably via Src family kinases (Palmer et al., 2002; Essmann et al., 2008). Evidence supporting this idea shows that phospho-Src is significantly increased after conditioning (data not shown), which may be responsible for the phosphorylated tyrosine and serine residues of ephrin-B1 after conditioning and BDNF treatment shown here. Moreover, phosphorylation of ephrin-B1 is blocked by postsynaptic injection of ephrin-B antibodies but not presynaptic injections. Forward EphB2 signaling is proposed to be induced by activation of postsynaptic ephrin-B, likely through ephrin-B1, but other ephrins cannot be excluded, to result in bouton growth. One caveat to this scheme, however, is that both EphB2 and ephrin-B1 are localized to the presynaptic and postsynaptic fractions (Fig. 7) even though ephrin-B1 predominates on the postsynaptic side. Therefore, there may be as yet undetected reverse or bidirectional interactions of Eph/ephrin that contribute to bouton growth during conditioning. Our findings show that neither conditioning nor BDNF treatment affects expression of phosphotyrosine and phosphoserine of EphB2, and consequently EphB2 appears to function through a mechanism that is independent of phosphorylation (Dalva et al., 2000; Kayser et al., 2006). Similar results were found by Grunwald et al. (2001) using BDNF application to cultured hippocampal neurons. If EphB2 is critical for ephrin-B-induced presynaptic modification as suggested here, why do levels of EphB2 phosphorylation remain unchanged? Instead, during conditioning, the C-terminal PDZ-binding motif of EphB2 may interact with PDZ scaffolding proteins involved in signal transduction for bouton growth, although there is currently little evidence for this occurring presynaptically. As with ephrin-B, different functions of the PDZ-binding motif and kinase domain of EphB have been demonstrated by removing or mutating particular segments. Kayser et al. (2006) showed that spine formation during development is controlled by the kinase domain of EphB2 while the PDZ-binding motif is required for AMPAR clustering. It is tempting to speculate that in conditioning, enhanced interaction of the PDZ-binding motif of EphB2 with other presynaptic interacting proteins may be required for presynaptic bouton growth. Conversely, phosphorylation of ephrin-B1 may additionally have an AMPAR trafficking role postsynaptically. We have already shown that there is a conditioning-related recruitment of GluR4-containing AMPAR synaptic localization in abducens motor neurons (Zheng and Keifer, 2009; Keifer and Zheng, 2010) that corresponds with presynaptic bouton growth. These coordinate presynaptic and postsynaptic actions would be expected to enhance overall synaptic function, resulting in $\mathrm{CR}$ acquisition ( $\mathrm{Li}$ and Keifer, 2008). Similarly, EphB/ephrin-B interactions were found to enhance presynaptic transmitter release, due at least in part to increased density in synapse number, and postsynaptic AMPAR responsiveness during reverse signaling in the developing Xenopus optic tectum (Lim et al., 2008). Therefore, Eph/ephrin signaling may be required for both synapse maturation and conditioning by retrograde structural modifications to enhance presynaptic function and by anterograde postsynaptic actions involving delivery of synaptic AMPARs as suggested by Lim et al. (2008) and the present study.

Here, we show a regulatory role for BDNF in ephrin-B phosphorylation. TrkB has also been shown to interact with ephrin family proteins via their cysteine-rich domains (Marler et al., 2008). We postulate (Fig. 7) that BDNF released during conditioning binds TrkB, leading to activation of postsynaptic ephrin-
B1. However, ephrin-B1 was also observed in presynaptic fractions, and we have found that TrkB is present in both presynaptic and postsynaptic fractions after differential centrifugation. If BDNF/TrkB functions mainly postsynaptically in conditioning, what may account for this specificity of function? Our preliminary data suggest that an $\sim 95 \mathrm{kDa}$ truncated version of TrkB is localized to presynaptic fractions, while the $\sim 145 \mathrm{kDa}$ fulllength TrkB is postsynaptic (data not shown). Truncated TrkB lacks the C-terminal kinase domain and is predicted to result in inhibition of BDNF function. Timed expression of truncated TrkB has been linked to reduced BDNF/TrkB activity and climbing fiber synapse elimination in cerebellar development (Sherrard et al., 2009). It is plausible that BDNF function during conditioning is restricted to postsynaptic targets by activitydependent presynaptic targeting of truncated rather than fulllength TrkB. The interaction between BDNF/TrkB function and the bidirectional Eph/ephrin signaling system requires further study to clarify their precise roles in coordinate presynaptic and postsynaptic mechanisms that underlie classical conditioning.

\section{References}

An JJ, Gharami K, Liao GY, Woo NH, Lau AG, Vanevski F, Torre ER, Jones KR, Feng Y, Lu B, Xu B (2008) Distinct role of long 3' UTR BDNF mRNA in spine morphology and synaptic plasticity in hippocampal neurons. Cell 134:175-187.

Anderson CW, Keifer J (1999) Properties of conditioned abducens nerve responses in a highly reduced in vitro brain stem preparation from the turtle. J Neurophysiol 81:1242-1250.

Becker N, Wierenga CJ, Fonseca R, Bonhoeffer T, Nägerl UV (2008) LTD induction causes morphological changes of presynaptic boutons and reduces their contacts with spines. Neuron 60:590-597.

Cohen-Cory S, Kidane AH, Shirkey NJ, Marshak S (2010) Brain-derived neurotrophic factor and the development of structural neuronal connectivity. Dev Neurobiol 70:271-288.

Contractor A, Rogers C, Maron C, Henkemeyer M, Swanson GT, Heinemann SF (2002) Trans-synaptic Eph receptor-ephrin signaling in hippocampal mossy fiber LTP. Science 296:1864-1869.

Dalva MB, Takasu MA, Lin MZ, Shamah SM, Hu L, Gale NW, Greenberg ME (2000) EphB receptors interact with NMDA receptors and regulate excitatory synapse formation. Cell 103:945-956.

De Roo M, Klauser P, Muller D (2008) LTP promotes a selective long-term stabilization and clustering of dendritic spines. PLoS Biol 6:e219.

Essmann CL, Martinez E, Geiger JC, Zimmer M, Traut MH, Stein V, Klein R, Acker-Palmer A (2008) Serine phosphorylation of ephrinB2 regulates trafficking of synaptic AMPA receptors. Nat Neurosci 11:1035-1043.

Geinisman Y, Disterhoft JF, Gundersen HJ, McEchron MD, Persina IS, Power JM, van der Zee EA, West MJ (2000) Remodeling of hippocampal synapses after hippocampus-dependent associative learning. J Comp Neurol 417:49-59.

Geinisman Y, Berry RW, Disterhoft JF, Power JM, Van der Zee EA (2001) Associative learning elicits the formation of multiple-synapse boutons. J Neurosci 21:5568-5573.

Grunwald IC, Korte M, Wolfer D, Wilkinson GA, Unsicker K, Lipp HP, Bonhoeffer T, Klein R (2001) Kinase-independent requirement of ephB2 receptors in hippocampal synaptic plasticity. Neuron 32:1027-1040.

Holtmaat A, Svoboda K (2009) Experience-dependent structural synaptic plasticity in the mammalian brain. Nat Rev Neurosci 10:647-658.

Holtmaat A, Wilbrecht L, Knott GW, Welker E, Svoboda K (2006) Experience-dependent and cell-type-specific spine growth in the neocortex. Nature 441:979-983.

Kayser MS, McClelland AC, Hughes EG, Dalva MB (2006) Intracellular and trans-synaptic regulation of glutamatergic synaptogenesis by ephB receptors. J Neurosci 26:12152-12164.

Keifer J, Mokin M (2004) Distribution of anterogradely labeled trigeminal and auditory nerve boutons on abducens motor neurons in turtles: implications for in vitro classical conditioning. J Comp Neurol 471:144-152.

Keifer J, Zheng Z (2010) AMPA receptor trafficking and learning. Eur J Neurosci 32:269-277. 
Keifer J, Sabirzhanov BE, Zheng Z, Li W, Clark TG (2009) Cleavage of proBDNF to BDNF by a tolloid-like metalloproteinase is required for acquisition of in vitro eyeblink classical conditioning. J Neurosci 29:14956-14964.

Klein R (2009) Bidirectional modulation of synaptic functions by Eph/ephrin signaling. Nat Neurosci 12:15-20.

Lai K-O, Ip NY (2009) Synapse development and plasticity: roles of ephrin/ Eph receptor signaling. Curr Opin Neurobiol 19:275-283.

Lee R, Kermani P, Teng KK, Hempstead BL (2001) Regulation of cell survival by secreted proneurotrophins. Science 294:1945-1948.

Li W, Keifer J (2008) Coordinate action of pre- and postsynaptic brainderived neurotrophic factor is required for AMPAR trafficking and acquisition of in vitro classical conditioning. Neuroscience 155:686-697.

Li W, Keifer J (2009) BDNF-induced synaptic delivery of AMPAR subunits is differentially dependent on NMDA receptors and requires ERK. Neurobiol Learn Mem 91:243-249.

Lim BK, Matsuda N, Poo MM (2008) Ephrin-B reverse signaling promotes structural and functional synaptic maturation in vivo. Nat Neurosci 11:160-169.

Lu B, Pang PT, Woo NH (2005) The yin and yang of neurotrophin action. Nat Rev Neurosci 6:603-614.

Marler KJ, Becker-Barroso E, Martínez A, Llovera M, Wentzel C, Poopalasundaram S, Hindges R, Soriano E, Comella J, Drescher U (2008) A TrkB/ephrinA interaction controls retinal axon branching and synaptogenesis. J Neurosci 28:12700-12712.

Moeller ML, Shi Y, Reichardt LF, Ethell IM (2006) EphB receptors regulate dendritic spine morphogenesis through the recruitment/phosphorylation of focal adhesion kinase and RhoA activation. J Biol Chem 281:1587-1598

Palmer A, Zimmer M, Erdmann KS, Eulenburg V, Porthin A, Heumann R,
Deutsch U, Klein R (2002) EphrinB phosphorylation and reverse signaling: regulation by $\mathrm{Src}$ kinases and PTP-BL phosphatase. Mol Cell 9:725-737.

Pang PT, Teng HK, Zaitsev E, Woo NT, Sakata K, Zhen S, Teng KK, Yung W-H, Hempstead BL, Lu B (2004) Cleavage of proBDNF by tPA/plasmin is essential for long-term hippocampal plasticity. Science 306: 487-491.

Sherrard RM, Dixon KJ, Bakouche J, Rodger J, Lemaigre-Dubreuil Y, Mariani J (2009) Differential expression of TrkB isoforms switches climbing fiberPurkinje cell synaptogenesis to selective synapse elimination. Dev Neurobiol 69:647-662.

Tanaka J, Horiike Y, Matsuzaki M, Miyazaki T, Ellis-Davies GC, Kasai H (2008) Protein synthesis and neurotrophin-dependent structural plasticity of single dendritic spines. Science 319:1683-1687.

Yang J, Siao CJ, Nagappan G, Marinic T, Jing D, McGrath K, Chen ZY, Mark W, Tessarollo L, Lee FS, Lu B, Hempstead BL (2009) Neuronal release of proBDNF. Nat Neurosci 12:113-115.

Yang Y, Wang XB, Frerking M, Zhou Q (2008) Spine expansion and stabilization associated with long-term potentiation. J Neurosci 28: $5740-5751$.

Zheng Z, Keifer J (2009) PKA has a critical role in synaptic delivery of GluR1- and GluR4-containing AMPARs during initial stages of acquisition of in vitro classical conditioning. J Neurophysiol 101:2539-2549.

Zhou L, Martinez SJ, Haber M, Jones EV, Bouvier D, Doucet G, Corera AT, Fon EA, Zisch AH, Murai KK (2007) EphA4 signaling regulates phospholipase $\mathrm{C} \gamma \mathrm{l}$ activation, cofilin membrane association, and dendritic spine morphology. J Neurosci 27:5127-5138.

Zhou Q, Homma KJ, Poo MM (2004) Shrinkage of dendritic spines associated with long-term depression of hippocampal synapses. Neuron 44: 749-757. 